\title{
A Subspace Module Extraction Technique for Gene Expression Data
}

\author{
Priyakshi Mahanta $^{1}$, Dhruba Kr. Bhattacharyya ${ }^{1}$, and Ashish Ghosh ${ }^{2}$ \\ 1 Department of Comp. Sc. and Engg., Tezpur University, Napaam -784028, India \\ 2 Machine Intelligent Unit, Indian Statistical Institute, Kolkata-700108, India \\ \{priyakshi, dkb\}@tezu.ernet.in, \\ ash@isical.ac.in
}

\begin{abstract}
Construction of co-expression network and extraction of network modules have been an appealing area of bioinformatics research. In literature, most existing algorithms of gene co-expression network extract network modules where all samples are considered. In this paper, we propose a method to construct a co-expression network based on mutual information and to extract network modules defined over a subset of samples. The method was applied over several real life gene expression datasets and the results are validated in terms of $\mathrm{p}$ value, $\mathrm{Q}$ value and topological properties.
\end{abstract}

Keywords: Co-expression network, network modules, mutual information, topological property.

\section{Introduction}

Mutual information is a measure of similarity between two variables. It has been widely used in various application domains including computational biology, machine learning, statistics, image processing, and financial computing. Mutual information (MI) provides a general measurement for dependencies in the data, in particular positive, negative and nonlinear correlations between two variables. An advantage of MI compared to many other similarity measures such as Pearson correlation and Euclidian distance, is its ability to detect non-linear correlations between two variables.

The concept of mutual information was initially developed for discrete data. The Mutual Information(MI) between two continuous random variable $X$ and $Y$ is defined as follows:

$$
I(X ; Y)=\iint f(x, y) \log \frac{f(x, y)}{f(x) f(y)} \mathrm{d} x \mathrm{~d} y .
$$

where $f(x, y)$ is the joint probability density of the two random variables and $f(x)$ and $f(y)$ are the marginal densities. Computing MI value for all possible pairs of genes is a computationally expensive task. In this paper, we use the 
method proposed by [1] where the order of calculations is rearranged so that repeating operations are avoided significantly.

Mutual Information between two variables can be computed if we have explicit knowledge of the joint probability distribution and marginal probability distribution. Generally these probabilities are not known. Various methods are used to estimate the probability densities from the observed data. Histogram approach and Kernel Estimation 2] are two popular methods used to estimate probabilities from observed data. Histogram approach is sensitive to the choice of the origin. Kernel estimation gives a better mean square error rate compared to histogram approach. Given $M$ data points drawn from the joint probability distribution, $\left(x_{j}, y_{j}\right)$ where $j=1,2,3, \ldots, M$, the joint and marginal probability can be estimated by gaussian kernel estimator where $h$ is a tuning parameter that determines the width of the kernel.

$$
\begin{gathered}
f(x, y)=\frac{1}{M} \sum \frac{1}{2 \pi h^{2}} e^{-\frac{1}{2 h^{2}}\left(x-x_{j}\right)^{2}+\left(y-y_{j}\right)^{2}} . \\
f(x)=\frac{1}{M} \sum \frac{1}{\sqrt{2 \pi h^{2}}} e^{-\frac{1}{2 h^{2}}\left(x-x_{j}\right)^{2}} . \\
f(y)=\frac{1}{M} \sum \frac{1}{\sqrt{2 \pi h^{2}}} e^{-\frac{1}{2 h^{2}}\left(y-y_{j}\right)^{2}} .
\end{gathered}
$$

Using Gaussian kernel, mutual information between two random variables $X$ and $Y$ can be computed as

$$
f(x, y)=\frac{1}{M} \sum_{i} \log \frac{M \sum_{j} e^{\left.-\frac{1}{2 h^{2}}\left(\left(x-x_{j}\right)^{2}\right)+\left(y-y_{j}\right)^{2}\right)}}{\sum_{j} e^{\left(-\frac{1}{2 h^{2}}\right)\left(x-x_{j}\right)^{2}} \sum_{j} e^{\left(-\frac{1}{2 h^{2}}\right)\left(y-y_{j}\right)^{2}}} .
$$

In this work, we have attempted to propose a method MIMET (Mutual Information based Module Extraction technique) to construct co-expression network that is capable of reflecting non-liner correlation among genes over subspaces of samples and to extract network modules, thereof.

\section{Method}

The method consists of two steps. In the first step, it extract network modules from the co-expression network based on mutual information. In the second step, subspace network modules are extracted using a discretization [3] process.

\subsection{Extraction of Network Modules}

Co-expression network is constructed by computing kernal based MI of each pair of genes. For each pair of genes, we need an appropriate value of $h$, a user defined parameter. Computing $h$ for each pair of genes is a time consuming process. Therefore, we propose a way to compute $h$ which works for all pairs of genes. To compute $h$, we first compute standard deviation of each gene considering all 
conditions in the gene expression dataset. Finally, $h$ is computed as standard deviation of the deviations of all genes. While constructing a co-expression network, say $G$ based on $D$, the proposed technique initially computes the adjacency matrix $A d j$ by using a soft thresholding approach. It encodes edge information for each pair of nodes in the co-expression network from the distance matrix. The distance matrix is obtained by using MI. The maximum spanning tree is extracted from the adjacency matrix using Prims algorithm [4] with weights defined by mutual information. The weakest edge in the spanning tree in terms of weight is removed to break the tree into two parts or subtrees. Each subtree is further processed to extract finer (i.e. strongly connected) module.

\subsection{Extraction of Subspace Network Modules}

In traditional network modules, the edges are defined over the whole set of samples. The subspace network modules are defined over subset of samples [5]. Each of the initial network module is processed individually to extract the subspace network modules. For each condition $c_{i}$, it identifies three subsets of genes: (i) subset with larger expression level, (ii) subset of genes with smaller expression level and (iii) subset of genes with similar expression level for condition $c_{i+1}$ with respect to condition $c_{i}$. Next it propagates through rest of conditions to filter the subsets in terms of regulation patterns with respect to selected conditions by including more conditions to finally form the subspace modules. If a subset of genes found in the earlier sub-step is unique with respect to it's expression pattern over the subset of conditions (i.e. not found in previous step), accept this subset of genes as a discovered set of subspace network modules which fulfils the criteria of minimum number of genes in a subspace module.

The complexity of finding MI matrix is $\mathrm{O}\left(\mathrm{n}^{2} m^{2}\right)$ where $n$ and $m$ are the number of gens and conditions in a dataset. Finding a maximum spanning tree consumes a complexity of $\mathrm{O}\left(n_{c} g_{a}^{2}\right)$ where $n_{c}$ is the number of network modules and $g_{a}$ is the average number of genes in the module. The complexity of finding subspace network modules is approximately of $\mathrm{O}\left(g_{s u b}\right)$ where $g_{s u b}$ is filtered average number of genes in terms of selected conditions in a module.

\section{$3 \quad$ Experimental Results}

We implemented MIMET algorithm in MATLAB and tested it on three benchmark microarray datasets mentioned in Table 1. The test platform was a Sun workstation with $\operatorname{Intel}(\mathrm{R}) \operatorname{Xenon}(\mathrm{R}) 3.33 \mathrm{GHz}$ processor and $6 \mathrm{~GB}$ memory running Windows XP operating system.

\subsection{Validation}

For biological validation of the extracted network modules, we used well known $\mathrm{p}$ and $\mathrm{Q}$ value. Also to establish effectiveness of MIMET, we used topological validation. 
Table 1. Datasets used for evaluating $M I M E T$

\begin{tabular}{|c|c|c|c|}
\hline $\begin{array}{c}\text { Serial. } \\
\text { No }\end{array}$ & Dataset & $\begin{array}{c}\text { No. of Genes/ } \\
\text { No. of Conditions }\end{array}$ & Source \\
\hline \hline 1 & Yeast Sporulation & $474 / 17$ & http://cmgm.standford.edu/pbrown/sporulation \\
\hline 2 & Human Dataset & $5008 / 4$ & Sample gene in expander \\
\hline 3 & Rat CNS & $112 / 9$ & http://faculty. washington.edu/kayee/cluster \\
\hline
\end{tabular}

(a) p value: Biological significance of the sets of genes included in the extracted network modules are evaluated based on p values [6] which signifies how well these genes match with different Gene Ontology(GO) categories. To compute p-value, we used a tool called FuncAssociate [7]. (b) Q value: Q-value [8] for

Table 2. p-values of a network module extracted for Dataset 1

\begin{tabular}{|c|l|l|}
\hline P-value & GO number & GO category \\
\hline $\mathbf{3 . 3 8 e - 3 1}$ & GO:0048646 & anatomical structure formation involved in morphogenesis \\
$\mathbf{5 . 8 9 e - 2 7}$ & GO:0042244 & spore wall assembly \\
$3.13 \mathrm{e}-23$ & GO:0048869 & cellular developmental process \\
$3.81 \mathrm{e}-20$ & GO:0070882 & cellular cell wall organization or biogenesis \\
$3.81 \mathrm{e}-20$ & GO:0071554 & cell wall organization or biogenesis \\
$1.06 \mathrm{e}-25$ & GO:0043934 & sporulation \\
$\mathbf{6 . 1 0 e - 2 9}$ & GO:0010927 & cellular component assembly involved in morphogenesis \\
$1.06 \mathrm{e}-25$ & GO:0030154 & cell differentiation \\
$\mathbf{3 . 5 3 e - 2 8}$ & GO:0070726 & cell wall assembly \\
$5.89 \mathrm{e}-27$ & GO:0030476 & ascospore wall assembly \\
$5.72 \mathrm{e}-16$ & GO:0005628 & prospore membrane \\
$\mathbf{5 . 8 9 e - 2 7}$ & GO:0071940 & fungal-type cell wall assembly \\
$2.06 \mathrm{e}-22$ & GO:0032502 & developmental process \\
$2.84 \mathrm{e}-17$ & GO:0048610 & cellular process involved in reproduction \\
$1.06 \mathrm{e}-15$ & GO:0006094 & gluconeogenesis \\
$6.60 \mathrm{e}-19$ & GO:0070882 & cellular cell wall organization or biogenesis \\
$\mathbf{1 . 4 3 e - 2 5}$ & GO:0030435 & sporulation resulting in formation of a cellular spore \\
\hline
\end{tabular}

a particular gene $G$ is the proportion of false positives among all genes which are more differentially expressed. We used a web based tool GeneMANIA [9] to generate hypotheses about gene function, analyzing gene lists and prioritizing genes for functional assays. From the given $\mathrm{p}$ and $\mathrm{Q}$ values shown in Tables 2 and 3 we can conclude that MIMET shows a good enrichment of functional categories and therefore project a good biological significance.

Topological Validation. The meaning of the edges in a gene co-expression network is a relevant question in network analysis. Different structural properties of co-expression network can be a solution to the above question. Therefore, in this paper the structural properties of the co-expression network inferred from gene expression microarray data are compared with topological properties of the known, well established network data of the same organism. We use a web based tool called topoGSA [10] for topological validation of the extracted network modules. MIMET has been found capable of identifying gene modules with very high average score $(\geq \mathbf{. 8 5})$ as shown in Table 4 . 
Table 3. Q-value of one of the network modules of Dataset 3

\begin{tabular}{|l|c|}
\hline$G O$ annotation & $Q$ value \\
\hline regulation of transmission of nerve impulse & $2.50 \mathrm{E}-11$ \\
axonogenesis & $2.50 \mathrm{E}-11$ \\
regulation of synaptic transmission & $2.50 \mathrm{E}-11$ \\
regulation of neuron apoptosis & $2.50 \mathrm{E}-11$ \\
axon & $2.50 \mathrm{E}-11$ \\
regulation of neurological system process & $4.20 \mathrm{E}-11$ \\
neuron apoptosis & $4.20 \mathrm{E}-11$ \\
neuron death & $5.33 \mathrm{E}-11$ \\
negative regulation of neuron apoptosis & $1.56 \mathrm{E}-10$ \\
transmembrane receptor protein tyrosine & \\
kinase signaling pathway & $2.13 \mathrm{E}-10$ \\
growth factor binding & $4.40 \mathrm{E}-9$ \\
protein autophosphorylation & $1.73 \mathrm{E}-8$ \\
\hline
\end{tabular}

Table 4. Ranking of KEGG gene sets based on topological similarity to uploaded module for dataset 2

\begin{tabular}{|c|c|l|c|c|l|l|}
\hline Identifier & $\begin{array}{c}\text { Median } \\
\text { degree }\end{array}$ & $\begin{array}{l}\text { Median } \\
\text { CC }\end{array}$ & $\begin{array}{c}\text { Median } \\
\text { SPL }\end{array}$ & $\begin{array}{c}\text { Median } \\
\text { BW }\end{array}$ & $\begin{array}{l}\text { Median } \\
\text { EVC }\end{array}$ & Score \\
\hline hsa05216: Thyroid cancer & 20 & 0.07 & 3.45 & 26256 & 0.05 & 0.83 \\
hsa05210: Colorectal cancer & 22.5 & 0.07 & 3.44 & 31963 & 0.05 & 0.84 \\
hsa04520:Adherens junction & 26 & 0.05 & 3.38 & 33514 & 0.07 & 0.85 \\
hsa04012:ErbB signaling pathway & 24 & 0.07 & 3.39 & 28589 & 0.07 & 0.87 \\
hsa05214:Glioma & 28 & 0.05 & 3.35 & 37425 & 0.07 & 0.87 \\
hsa05213:Endometrial cancer & 24 & 0.07 & 3.38 & 33066 & 0.07 & 0.87 \\
hsa01510:Neurodegenerative Diseases & 33 & 0.04 & 3.33 & 67612 & 0.05 & 0.88 \\
hsa05215:Prostate cancer & 28 & 0.06 & 3.37 & 35660 & 0.07 & 0.89 \\
hsa05221:Acute myeloid leukemia & 27 & 0.09 & 3.39 & 32521 & 0.07 & 0.9 \\
hsa04320:Dorso-ventral axis formation & 30 & 0.06 & 3.32 & 32814 & 0.1 & 0.91 \\
hsa05223:Non-small cell lung cancer & 29 & 0.07 & 3.35 & 38253 & 0.08 & 0.91 \\
hsa05212:Pancreatic cancer & 30 & 0.07 & 3.35 & 45788 & 0.07 & 0.92 \\
hsa05220:Chronic myeloid leukemia & 36 & 0.08 & 3.3 & 49218 & 0.1 & 0.96 \\
\hline
\end{tabular}

\section{Conclusion and Future Work}

In this paper, a gene co-expression network construction technique called MIMET based on a soft thresholding approach is presented. The performance of MIMET is excellent in three publicly available benchmark real life datasets. From the network, subspace network modules are extracted using a discretization process. An adaptive discretization technique can also be used to extract more biologically effective network modules. Construction of gene regulatory network from co-expression network with the support of Gene Ontology can lead to better, more meaningful predictions of gene networks.

Acknowledgment. This paper is an outcome of a research project supported by DST, Govt. of India in collaboration with CSCR, ISI, Kolkata.

\section{References}

[1] Qiu, P., Gentles, A.J., Plevritis, S.K.: Fast calculation of pairwise mutual information for gene regulatory network reconstruction. Comput. Methods Prog. Biomed. 94(2), 177-180 (2009) 
[2] Moon, Y.I., Rajagopalan, B., Lall, U.: Estimation of mutual information using kernel density estimators. Phys. Rev. E 52(3) (September 1995)

[3] Mahanta, P., Ahmed, H.A., Kalita, J.K., Bhattacharyya, D.K.: Discretization in gene expression data analysis: a selected survey. In: Proceedings of the Second International Conference on Computational Science, Engineering and Information Technology, CCSEIT 2012, pp. 69-75. ACM, New York (2012)

[4] Prim, R.C.: Shortest connection networks and some generalizations. Bell System Technology Journal 36, 1389-1401 (1957)

[5] Sharma, S., Bhattacharyya, D.K.: An effective technique for clustering incremental gene expression data. International Journal of Computer Science Issues 7 (2010)

[6] Tavazoie, S., Hughes, J.D., Campbell, M.J., Cho, R.J., Church, G.M.: Systematic determination of genetic network architecture. Nature Genetics (1999)

[7] Berriz, G.F., King, O.D., Bryant, B., Sander, C., Roth, F.P.: Characterizing gene sets with FuncAssociate. Bioinformatics (Oxford, England) 19, 2502-2504 (2003)

[8] Benjamini, Y., Hochberg, Y.: Controlling the false discovery rate: A practical and powerful approach to multiple testing. Journal of the Royal Statistical Society. Series B (Methodological) 57, 289-300 (1995)

[9] Warde-Farley, D., Donaldson, S.L., Comes, O., Zuberi, K., Badrawi, R., Chao, P., Franz, M., Grouios, C., Kazi, F., Lopes, C.T., Maitland, A., Mostafavi, S., Montojo, J., Shao, Q., Wright, G., Bader, G.D., Morris, Q.: The GeneMANIA prediction server: biological network integration for gene prioritization and predicting gene function. Nucleic Acids Research 38, W214-W220 (2010)

[10] Glaab, E., Baudot, A., Krasnogor, N., Valencia, A.: TopoGSA: network topological gene set analysis. Bioinformatics 26(9), 1271-1272 (2010) 